\title{
Applications of the $g$-Drazin Inverse to the Heat Equation and a Delay Differential Equation
}

\author{
Alrazi Abdeljabbar and Trung Dinh Tran \\ Department of Mathematics, College of Arts and Sciences, Khalifa University for Science and Technology-The Petroleum Institute, \\ P. O. Box 2533, Abu Dhabi, UAE
}

Correspondence should be addressed to Alrazi Abdeljabbar; aabdeljabbar@pi.ac.ae

Received 11 June 2017; Accepted 11 October 2017; Published 1 November 2017

Academic Editor: Carlos Lizama

Copyright (c) 2017 Alrazi Abdeljabbar and Trung Dinh Tran. This is an open access article distributed under the Creative Commons Attribution License, which permits unrestricted use, distribution, and reproduction in any medium, provided the original work is properly cited.

We consider applications of the $g$-Drazin inverse to some classes of abstract Cauchy problems, namely, the heat equation with operator coefficient and delay differential equations in Banach space.

\section{Introduction}

In this paper we utilize the generalized Drazin inverse for closed linear operators to obtain explicit solutions to two types of abstract Cauchy problem. The first type is the heat equation with operator coefficient. The second type is a delay differential equation.

Firstly let us consider the heat equation with operator coefficient. Let $A$ be a bounded linear operator in a Hilbert space $X$ and $g$ be a holomorphic $X$-valued function. The following initial value problem

$$
\begin{aligned}
\frac{\partial u(t, x)}{\partial t} & =A \frac{\partial^{2} u(t, x)}{\partial x^{2}} \\
u(0, x) & =g(x)
\end{aligned}
$$

is studied in [1] under the assumption that $A$ is a Volterra operator and its imaginary part of $A$ is of trace class. In particular, it has been proved that if $A$ is quasinilpotent and its imaginary part $A_{I}:=(1 / 2 i)\left(A-A^{*}\right)$ is of trace class, then the Cauchy problem has a unique holomorphic solution in a neighborhood of zero.

We study the above Cauchy problem for the case where $A$ is a positive operator, and 0 is not an accumulated spectral point of $A$. Our results are extensions of [1] in the sense that the class of $g$-Drazin invertible operators $A$ is more general than that of quasinilpotent operators.
We will show that if $A$ is positive and $g$-Drazin invertible then the solution to the system

$$
\begin{aligned}
\frac{\partial u(t, x)}{\partial t} & =A \frac{\partial^{2} u(t, x)}{\partial x^{2}} \\
\lim _{t \rightarrow 0^{+}} u(t, x) & =g(x)
\end{aligned}
$$

exists and is given by an explicit formula. We say a function $u(t, x)$ is a solution to the above initial value problem if it satisfies the partial differential equation in $[0, T) \times \mathbb{R}$ for some $T>0$, and $\lim _{t \rightarrow 0^{+}} u(0, x)=g(x)$ with $g(x)$ being an analytic function satisfying the bounds $\|g(x)\| \leq a e^{b x^{2}}$, where $a$ and $b$ are some positive constants.

Secondly we consider the following delay differential equation

$$
y^{\prime}(z)=A y(z-h)+f(z)
$$

in a Banach space $X$, which is studied by Gefter and Stulova in [2] under the assumption that $A$ is an invertible closed linear operator with a bounded inverse in $X$; the delay term $h$ is a complex constant, and $f$ is an $X$-valued holomorphic function of zero exponential type. Recall that an entire function $f$ is of zero exponential type if, for every $\epsilon>0$, there exists $C_{\epsilon}>0$ such that $\|f(z)\| \leq C_{\epsilon} e^{\epsilon|z|}$ for each $z \in \mathbb{C}$. We generalize the results in [2] by replacing the invertible closed linear operator $A$ with a $g$-Drazin invertible operator. We 
will show that if $A$ is $g$-Drazin invertible and $f$ is an entire function of zero exponential type, then the delay equation (3) has an entire solution of zero exponential type and it is expressed by an explicit formula.

Following [3], a closed linear operator $A$ is $g$-Drazin invertible if 0 is not an accumulated spectral point of $A$. By $\sigma(A), R(A), D(A)$, and $N$ we denote the spectrum, range, domain, and nullspace of $A$, respectively. A bounded linear operator $B$ is called a $g$-Drazin inverse of $A$ if $\mathscr{R}(B) \subset \mathscr{D}(A)$, $\mathscr{R}(I-A B) \subset \mathscr{D}(A)$, and

$$
\begin{aligned}
B A & =A B, \\
B A B & =B, \\
\sigma(A(I-A B)) & =\{0\} .
\end{aligned}
$$

Such an operator is unique, if it exists and is denoted by $A^{D}$. From [3], we have the following decomposition result.

Theorem 1. If $A$ is a g-Drazin invertible operator in a Banach space $X$, then $X=\mathscr{R}\left(A^{D} A\right) \oplus N\left(A^{D} A\right), A=A_{1} \oplus A_{2}$, where $A_{1}$ is closed and invertible, $A_{2}$ is bounded and quasinilpotent with respect to this direct sum, and

$$
A^{D}=A_{1}^{-1} \oplus 0 .
$$

Moreover, if $P$ is the spectral projection corresponding to 0 , then $P=I-A A^{D}$.

The above result is crucial to our analysis.

\section{Solution for the Heat Equation with Positive Operator Coefficient}

In this section we obtain an analytic solution for (2) that generalizes [1, Theorem 2] in the sense that the coefficient operator $A$ is assumed to be $g$-Drazin invertible instead of quasinilpotent.

Theorem 2. Let $A$ be a closed positive operator which is $g$ Drazin invertible, and let $g$ be an analytic function in $\mathbb{R}$ that satisfies the bound $\|g(x)\| \leq a e^{b x^{2}}$ for some positive constants $a$ and $b$. Then the system (2) has a unique solution given by the formula

$$
\begin{aligned}
& u(t, x) \\
& =P g(x)+\sum_{n=1}^{\infty} \frac{t^{n}}{n !} A^{n} P g^{(2 n)}(x) \\
& \quad+\frac{\left(A^{D}\right)^{1 / 2}}{\sqrt{4 \pi t}} \int_{-\infty}^{\infty} e^{-A^{D}(x-y)^{2} / 4 t}(I-P) g(y) d y,
\end{aligned}
$$

where $P=I-A A^{D}, e^{-A^{D} s}$ represents the $C_{0}$-semigroup of linear bounded operators generated by $-A^{D}$, and $\left(A^{D}\right)^{1 / 2}$ denotes a bounded operator $B$ such that $B^{2}(I-P)=A^{D}(I-P)$.

Proof. Since $A$ is $g$-Drazin invertible, by Theorem (1), $X=$ $\mathscr{R}(I-P) \oplus N(I-P), A=A_{1} \oplus A_{2}$, where $A_{1}$ is closed invertible and $A_{2}$ is bounded quasinilpotent with respect to the direct sum. Therefore Problem (2) has a unique solution if and only if each of the following two initial value problems has a unique solution on $\mathscr{R}(I-P)$ and $\mathscr{R}(P)$, respectively.

$$
\begin{aligned}
\frac{\partial u_{1}}{\partial t} & =A_{1} \frac{\partial^{2} u_{1}}{\partial x^{2}} \\
\lim _{t \rightarrow 0^{+}} u_{1}(0, x) & =(I-P) g(x), \\
\frac{\partial u_{2}}{\partial t} & =A_{2} \frac{\partial^{2} u_{2}}{\partial x^{2}} \\
\lim _{t \rightarrow 0^{+}} u_{2}(0, x) & =P g(x) .
\end{aligned}
$$

Since the operator $A$ is positive, it is self-adjoint. Therefore, $A_{2}$ is self-adjoint and the imaginary part of $A_{2}$ is zero. Applying [1, Theorem 2] to Problem (8),

$$
u_{2}(t, x)=P g(x)+\sum_{n=1}^{\infty} \frac{t^{n}}{n !} A_{2}^{n} P g^{(2 n)}(x)
$$

is the unique solution of Problem (8). Next we will show that

$$
\begin{aligned}
& u_{1}(t, x) \\
& \quad=\frac{\left(A_{1}^{-1}\right)^{1 / 2}}{\sqrt{4 \pi t}} \int_{-\infty}^{\infty} e^{-A_{1}^{-1}(x-y)^{2} / 4 t}(I-P) g(y) d y
\end{aligned}
$$

is the unique solution of Problem (7). The operator $\left(A_{1}^{-1}\right)^{1 / 2}$ denotes an operator $B$ such that $A_{1}^{-1}=B^{2}$. The existence of such an operator $B$ is guaranteed by the positivity of $A_{1}^{-1}$.

Since $A$ is positive, $\sigma(A) \subset[0, \infty)$, which implies $\sigma\left(-A_{1}^{-1}\right) \subset(-\infty, 0)$. Therefore, there exist constants $\mu>0$ and $M>0$ such that

$$
\begin{aligned}
& \left\|\int_{-\infty}^{\infty} e^{-A_{1}^{-1}(x-y)^{2} / 4 t}(I-P) g(y) d y\right\| \\
& \quad \leq \int_{-\infty}^{\infty} M e^{-\mu(x-y)^{2} / 4 t}\|I-P\|\|g(y)\| d y .
\end{aligned}
$$

Observe that the above inequality reduces the analysis of the heat equation with operator coefficient to that of the standard heat equation with scalar coefficient

$$
\begin{aligned}
\frac{\partial u(t, x)}{\partial t} & =\frac{1}{\mu} \frac{\partial^{2} u(t, x)}{\partial x^{2}} \\
u(0, x) & =g(x) .
\end{aligned}
$$

This allows us to apply standard results of the heat equation with scalar coefficient to Problem (7). In particular, using the last inequality, the bounds on $\|g(x)\|$, and the fundamental solution to the heat equation, one can differentiate under the integrals and verify that the integrals for $u_{1}, \partial u_{1} / \partial t$ and $\partial^{2} u_{1} / \partial x^{2}$ all converge. Using the derivative of the $C_{0}$ semigroup $d e^{-A_{1}^{-1} s} / d s=-A_{1}^{-1} e^{-A_{1}^{-1} s}$, it is straightforward to check that $u_{1}(t, x)$ satisfies the partial differential equation (7). Moreover, $u_{1}(t, x)$ is the only solution if $(x, t) \in[0$, $\mu / 4 b) \times \mathbb{R}$, and $\lim _{t \rightarrow 0^{+}} u_{1}(t, x)=(I-P) g(x)$. 
Since $A^{D}(I-P)=A_{1}^{-1}(I-P)$ and $A^{n} P=A_{2}^{n} P$, we obtain $u(t, x)$

$$
\begin{aligned}
&= P g(x)+\sum_{n=1}^{\infty} \frac{t^{n}}{n !} A^{n} P^{(2 n)}(x) \\
&+\frac{\left(A^{D}\right)^{1 / 2}}{\sqrt{4 \pi t}} \int_{-\infty}^{\infty} e^{-A^{D}(x-y)^{2} / 4 t}(I-P) g(y) d y, \\
& \lim _{t \rightarrow 0^{+}} u(t, x)=\lim _{t \rightarrow 0^{+}} u_{1}(t, x)+\lim _{t \rightarrow 0^{+}} u_{2}(t, x) \\
&=(I-P) g(x)+P g(x)=g(x) .
\end{aligned}
$$
taking

An application of the above result can be illustrated by

$$
A=-\frac{d^{2}}{d x^{2}}
$$

where

$$
\begin{aligned}
\mathscr{D} & (A)=\left\{w \in L^{2}(-\pi, \pi), A w \in L^{2}(-\pi, \pi), w(-\pi)\right. \\
= & \left.w(\pi), w^{\prime}(-\pi)=w^{\prime}(-\pi)\right\} .
\end{aligned}
$$

For more details about this operator we refer the reader to [4, page 389].

\section{Solution to the Delay Differential Equation}

In this section we obtain a holomorphic solution to the delay differential equation (3). The result generalizes [2, Theorem 2].

Theorem 3. Let $A$ be a closed linear operator which is $g$ Drazin invertible, and let $f$ be an entire function of zero exponential type. Then (3) has a zero exponential type solution given by the formula

$$
\begin{aligned}
y(z)= & -\sum_{n=0}^{\infty}\left(A^{D}\right)^{n+1}(I-P) f^{(n)}(z+(n+1) h) \\
& +\sum_{n=0}^{\infty} A^{n} P F^{(n+1)}(z-n h),
\end{aligned}
$$

where $P=I-A A^{D}$ and $F^{(n)}$ is the $n$-th primitive of $f$; that is, $d^{n} F^{(n)}(z) / d z^{n}=f(z)$.

Proof. Since $A$ is $g$-Drazin invertible, $X=\mathscr{R}(I-P) \oplus N(I-P)$, $A=A_{1} \oplus A_{2}$, where $A_{1}$ is closed and invertible and $A_{2}$ is bounded and quasinilpotent with respect to the direct sum. Therefore (3) has a solution if and only if each of the following two initial value problems has a solution on $\mathscr{R}(I-P)$ and $\mathscr{R}(P)$, respectively.

$$
\begin{aligned}
& y_{1}^{\prime}(z)=A_{1} y_{1}(z-h)+f_{1}(z), \\
& y_{2}^{\prime}(z)=A_{2} y_{2}(z-h)+f_{2}(z) .
\end{aligned}
$$

Since the operator $A_{1}$ is closed and invertible, applying [2, Theorem 2] to (17), we have

$$
y_{1}(z)=-\sum_{n=0}^{\infty} A_{1}^{-(n+1)} f_{1}^{(n)}(z+(n+1) h)
$$

being the unique solution of Problem (17). Next we will show that

$$
y_{2}(z)=\sum_{n=0}^{\infty} A_{2}^{n} F_{2}^{(n+1)}(z-n h)
$$

is a zero exponential type solution of Problem (18). Following [2, Lemma 1], we first show that if $f_{2}(z)$ is of zero exponential type then so is $F_{2}^{(n)}(z)$. Let $f_{2}(z)=\sum_{n=0}^{\infty} \alpha_{m} z^{m}$ be of zero exponential type and $\epsilon>0$. Since $\lim _{m \rightarrow \infty}\left(m !\left\|\alpha_{m}\right\|\right)^{1 / m}=0$ for each $m \in \mathbb{N},\left\|\alpha_{m}\right\| \leq M\left(\epsilon^{m} / m !\right)$ for some $M>0$. Letting $m+n=k$, we have

$$
\begin{aligned}
\left\|F_{2}^{(n)}(z)\right\| & =\left\|\sum_{m=0}^{\infty} \frac{\alpha_{m} z^{m+n}}{(m+1)(m+2) \cdots(m+n)}\right\| \\
& =\left\|\sum_{k=n}^{\infty} \alpha_{k-n} z^{k} \frac{(k-n) !}{k !}\right\| \leq \sum_{k=n}^{\infty} M \frac{\epsilon^{k-n}}{k !}|z|^{k} \\
& \leq \frac{M}{\epsilon^{n}} e^{\epsilon|z|} .
\end{aligned}
$$

Now, modifying the proof of [2, Theorem 1] with the $n$ th derivative replaced by the $n$-th primitive $F_{2}^{(n)}(z), \epsilon^{n}$ by $\epsilon^{-n}$ and $n h$ by $-n h$, we obtain the convergence of $y_{2}(z)$ and its sum is an entire function of zero exponential type. It is straightforward to check that the infinite sum is a solution of (18). Since $A^{D}(I-P)=A_{1}^{-1}(I-P)$ and $A^{n} P=A_{2}^{n} P$, we obtain

$$
\begin{aligned}
y(z)= & -\sum_{n=0}^{\infty}\left(A^{D}\right)^{n+1}(I-P) f^{(n)}(z+(n+1) h) \\
& +\sum_{n=0}^{\infty} A^{n} P F^{(n+1)}(z-n h) .
\end{aligned}
$$

\section{Conclusion}

In Section 2 we have obtained the unique solution for the heat equation with operator coefficient $A$, which is assumed to be self-adjoint and positive in a Hilbert space. Our result extends [1, Theorem 2] in the sense that $A$ is $g$-Drazin invertible instead of quasinilpotent. In Section 3 we have obtained an explicit solution for the delay differential equation with singular operator coefficient. Our result extends [2, Theorem 2 ] in the sense that $A$ is $g$-Drazin invertible instead of invertible in the usual sense.

\section{Conflicts of Interest}

The authors declare that they have no conflicts of interest. 


\section{References}

[1] S. Gefter and A. Vershynina, "On holomorphic solutions of the heat equation with a Volterra operator coefficient," Methods of Functional Analysis and Topology, vol. 13, no. 4, pp. 329-332, 2007.

[2] S. Gefter and T. Stulova, "On solutions of zero exponential type for some inhomogeneous differential-difference equations in a Banach space," in Progress and Challenges in Dynamical Systems, Springer Proceedings in Mathematics and Statistics, vol. 54 of Springer Proc. Math. Stat., pp. 253-263, Springer, Berlin, Germany, 2013.

[3] J. J. Koliha and T. D. Tran, "The Drazin inverse for closed linear operators and the asymptotic convergence of $C_{0}$-semigroups," The Journal of Operator Theory, vol. 46, no. 2, pp. 323-336, 2001.

[4] T. Katö, Perturbation Theory for Linear Operators, vol. 132 of Classics in Mathematics, Springer, Berlin, Germany, 1995. 


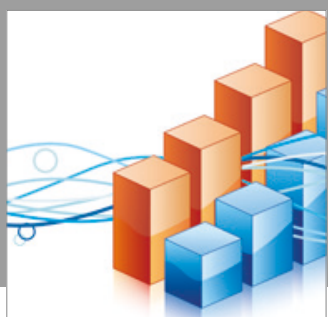

Advances in

Operations Research

vatersals

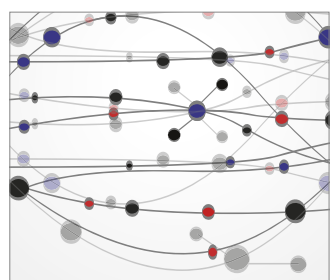

\section{The Scientific} World Journal
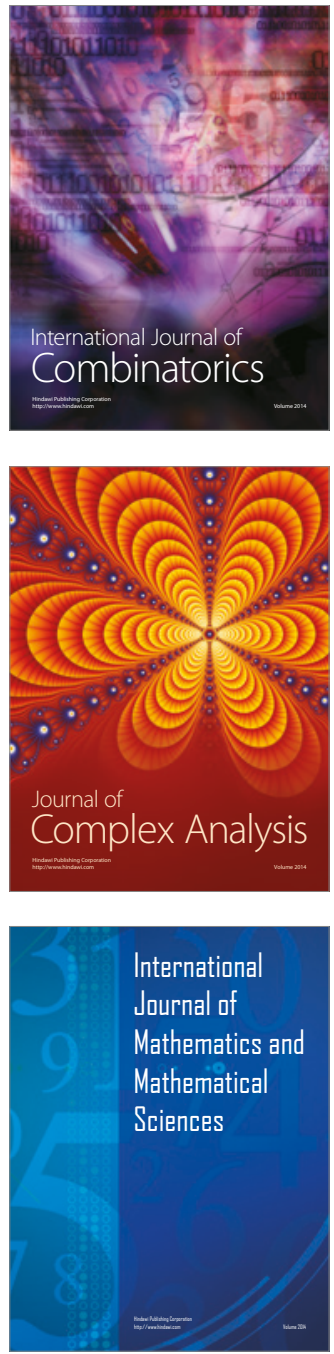
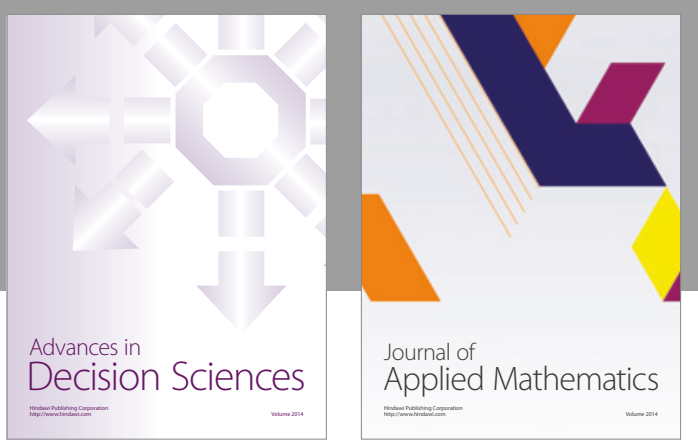

Algebra

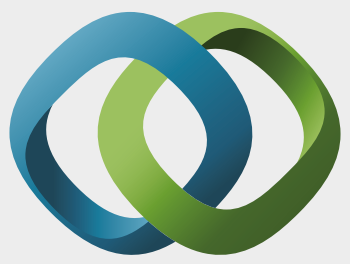

\section{Hindawi}

Submit your manuscripts at

https://www.hindawi.com
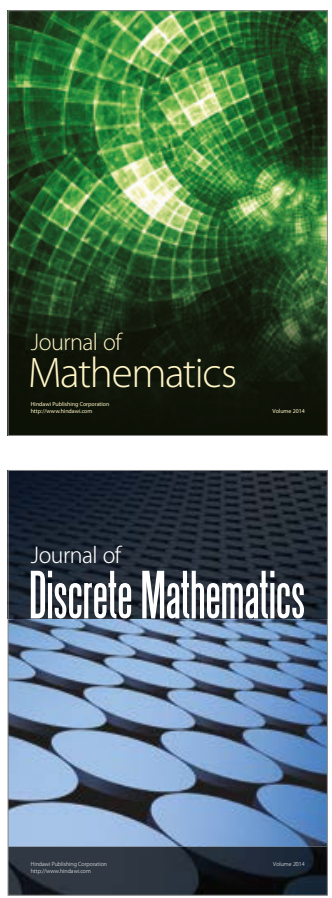

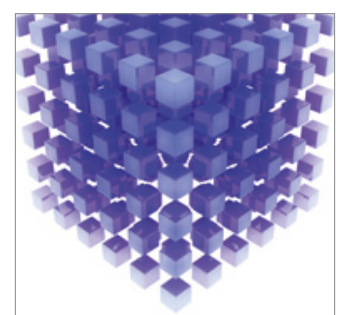

Mathematical Problems in Engineering
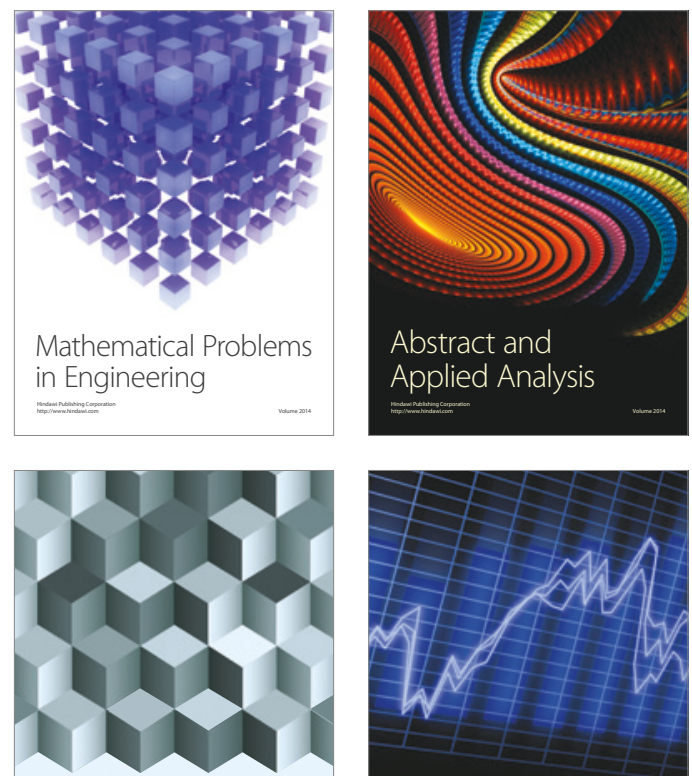

Journal of

Function Spaces

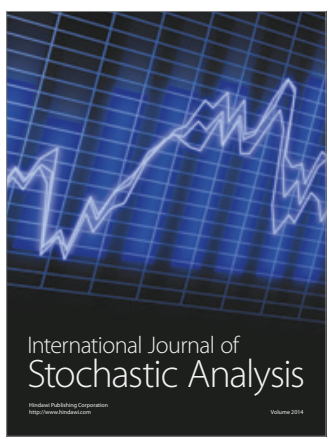

Probability and Statistics
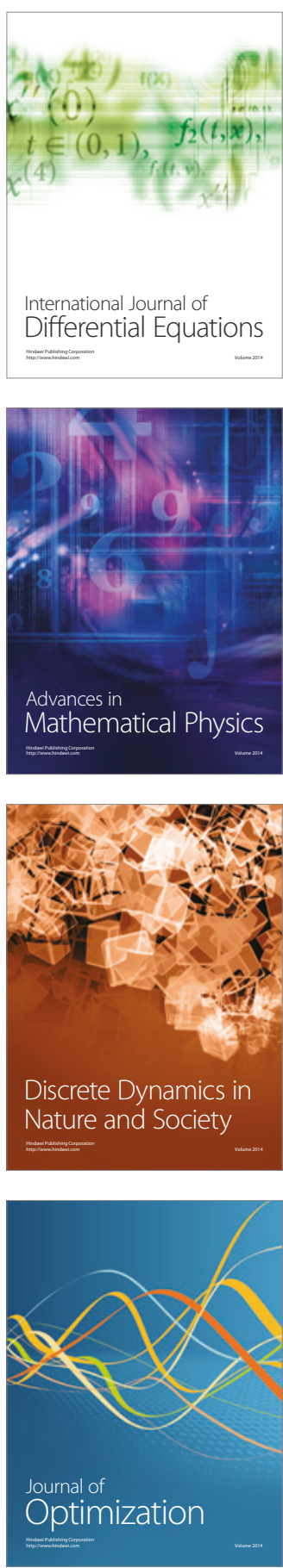